\title{
Prevalence and antibiogram of methicillin resistant Staphylococcus aureus isolated from medical device-related infections; a retrospective study in Lahore, Pakistan
}

\author{
Muhammad Sohail ${ }^{[1]}$ and Zakia Latif ${ }^{11]}$
}

[1]. Department of Microbiology and Molecular Genetics, University of the Punjab, Quaid-e-Azam Campus, Lahore-54590, Pakistan.

\begin{abstract}
Introduction: With the advancement of medicine and surgery, various types of medical devices have become part of treatment strategies. Methods: Identification and antimicrobial sensitivity testing were done according to CLSI guidelines following standard microbiological practices. Results: Urinary catheter infections (31\%) were most frequent followed by central venous catheter (18\%) and orthopedic implants (15\%). Methicillin resistant Staphylococcus aureus (MRSA) was a major cause of device-related infection after Escherichia coli (21\%); other pathogens were Klebsiella pneumoniae (14\%), Pseudomonas spp. (10\%), Acinetobacter spp. (8\%) and Candida species (7\%). None of MRSA was resistant to vancomycin (MIC $\geq 16 \mu \mathrm{g} / \mathrm{mL}$ ). Resistance rates were $98 \%$ and $97 \%$ for ofloxacin and ciprofloxacin, respectively. Conclusions: Escherichia coli and MRSA are major pathogens of medical device-related infections.
\end{abstract}

Keywords: Prosthetic devices. MRSA. Escherichia coli. Vancomycin. Antibiogram.

Healthcare-associated infection is a major problem of modern health care management; it is suggested that $60 \%$ of these infections are related to prosthetic devices. Advancements in medicine, surgery and bioengineering have paved the way for the use of prosthetic and medical devices in healthcare. This marvelous achievement proved a turning point in medicine that led to better treatment options. Patients with prosthetic devices, catheters or implants are more vulnerable to infections. Devicerelated infections are caused by Staphylococcus aureus and Staphylococcus epidermidis that have the potential for biofilm formation. This makes them difficult to eradicate even after removal of the prosthetic device and leads to chronic infection. Central venous catheters (CVCs) are essential devices used for medication and nutrition to critically ill patients. Infection of CVCs dramatically increases the morbidity (35\%), hospital stay and cost to the health care setting along with medical complications. Bacteremia is an outcome of CVC infections in newborn infants and the risk increases with the duration of hospital stay. CVC infections are the most (90\%) common cause of catheter-related infections and increase the mortality by 2.27 folds $^{1}$. Infection of catheters depends on multiple factors, including retention time, the procedure of catheterization,

Corresponding author: Muhammad Sohail.

e-mail: drsohailmmg@gmail.com

Received 31 August 2016

Accepted 30 June 2017 sterilization status of the healthcare setting, immunocompetence status of the patient, and nutritional status. Urinary tract infections (UTIs) are very common, and $80 \%$ of UTIs are due to urinary catheterization, which makes patients vulnerable to uropathogens. Catheterization is a common procedure (15-20\%) among hospitalized patients worldwide. Bacterial translocation is the main source of infection due to endotracheal intubation. The endotracheal tube (ETT) is an independent risk factor for developing ventilator-associated pneumonia (VAP); which is reported in $15-20 \%$ of mechanically ventilated patients. Catheter-related infection is the major complication and drawback of peritoneal dialysis, which is associated with a $3.5-10 \%$ mortality rate ${ }^{2}$. For the management of hydrocephalus, ventriculoperitoneal (VP) shunt is preferred to ventriculoatrial (VA) shunt because it is associated with fewer complications and is a relatively straightforward surgery. VA/VP shunt infections were reported as $4.2 \%$ to $11.3 \%$, which is very high for a complicated medical condition. The control of prosthetic and healthcare-associated infection is a challenge today, and there is an urgent need to take precautionary measures against it.

This study aimed to determine the prevalence and identification of bacteria causing indwelling device-related infections and to evaluate the antimicrobial sensitivity pattern of methicillin resistant Staphylococcus aureus (MRSA). Six major catheter or indwelling devices including CVCs, urinary catheters, ETT, peritoneal dialysis catheter, and VA/VP shunts were included in this study.

This study was conducted in the Department of Microbiology and Molecular Genetics, University of the Punjab, Lahore, 
Pakistan from March 2012 to March 2016. During this period, samples were taken from outpatients and in-patients with prosthetic device-related infection. The specimens included the prosthetic devices themselves, part of the prosthetic device, or the pus and urine from the infection site.

All the specimens were processed aseptically according to standard microbiological protocols. Pus specimens were inoculated on sheep blood agar, MacConkey and chocolate agar plates, whereas urine samples were inoculated on cystine lactose electrolyte deficient (CLED) agar plates. All inoculated plates were incubated overnight aerobically at $37 \mathrm{C}^{\circ}$. Prosthetic devices and catheters were incubated in brain heart infusion for two hours and then inoculated on agar plates to maximize the recovery of pathogens. The guidelines of the Clinical and Laboratory Standards Institute (CLSI) were followed to declare infection especially urine catheter and ETT infections.

Following the CLSI guidelines, antimicrobial activity of the isolates were determined by the disk diffusion method ${ }^{3}$. MRSA was confirmed by the cefoxitin interpretation criteria per the CLSI recommendations: $S$. aureus having an inhibition zone $\geq 22 \mathrm{~mm}$ was confirmed as MRSA. The minimum inhibitory concentration (MIC) of vancomycin was determined by the E-test strip (Etest ${ }^{\circledR}$ bioMérieux, USA) method by following the procedure of the manufacturer and interpretation criteria of the CLSI ${ }^{3}$.

Statistical analysis was conducted using Statistical Package for the Social Sciences (SPSS) version 20 (IBM Corp., Armonk, NY, USA). Chi square $\left(\chi^{2}\right)$ test was performed to analyze the difference among proportions of resistance in different years among samples with isolated MRSA.

Among 6,242 prosthetic device-related infection samples, $79 \%(4,902)$ were positive for bacterial growth. Among the infected patients $65 \%(3,186)$ were male and $35 \%(1,716)$ were female. The age of the participants ranged from 10 days to 80 years, with 44 being the median age for males and 33 years for females. Urinary catheter infections $(31 \%)$ were prominent among the device-related infections, followed by CVCs (18\%) and orthopedic implants (15\%). The $\chi^{2}$ analysis revealed that the number of patients with prosthetic device infection increased significantly with time $\left.\left[\chi^{2}(2 \mathrm{~N}=4,902)\right]=18, p=0.04\right)$. Overall, Escherichia coli (21\%) was most common cause of device-related infections followed by MRSA (15\%), Klebsiella pneumoniae (14\%), coagulase negative Staphylococcus (CONS) (11\%), Pseudomonas spp. (10\%), MSSA (10\%), Acinetobacter spp. (8\%), Candida spp. (7\%), and Streptococcus species (4\%) (Figure 1).

MRSA was most common pathogen after E. coli, isolated from patients with indwelling device-related infection. MRSA was isolated from $30 \%$ of orthopedic implants followed by CVCs (27\%), peritoneal dialysis catheters (14\%), VP/VA shunts $(8 \%)$, ETTs $(7 \%)$ and urinary catheters $(5 \%)$. Agewise prevalence of MRSA was determined among patients with prosthetic device-related infection. The most affected age group was $31-40$ years $(29.9 \%)$, followed by $41-50$ years $(23.3 \%)$ and $21-30$ years $(19.7 \%)$. The present study showed that prosthetic device infection was less $(4.5 \%)$ prevalent among individuals younger than 10 years of age.



FIGURE 1 - Prevalence of pathogens in device-related infections. VP/VA: ventriculoperitoneal/ventriculoatrial; MRSA: methicillin resistant Staphylococcus aureus; MSSA: methicillin-sensitive Staphylococcus aureus; CONS: coagulase negative Staphylococcus. 
The antibiogram of MRSA was determined with 14 antibiotics recommended by the CLSI ${ }^{3}$. Most of the MRSA isolated from prosthetic devices were resistant to ofloxacin (98\%), ciprofloxacin (97\%), followed by tobramycin (92\%), macrolides $(88 \%)$, and gentamycin $(87 \%)$; as shown in Table 1. Linezolid (1.1\% resistance) and vancomycin were the most effective against MRSA isolated from the patients with prosthetic device-related infections. The resistance pattern of MRSA isolated from the patients with prosthetic device-related infection is shown in Figure 2.

None of the MRSA was fully resistant to vancomycin (MIC $\geq 16 \mu \mathrm{g} / \mathrm{mL}$ ); $5.75 \%$ isolates had intermediate resistance (MIC, $4-8 \mu \mathrm{g} / \mathrm{mL})$. A total of $31.5 \%$ of MRSA were inhibited by $1 \mu \mathrm{g} / \mathrm{mL}$ of vancomycin and $25 \%$ by $0.5 \mu \mathrm{g} / \mathrm{mL}$ (Figure 2).

TABLE 1

Antibiogram of MRSA isolated from prosthetic device related infections.

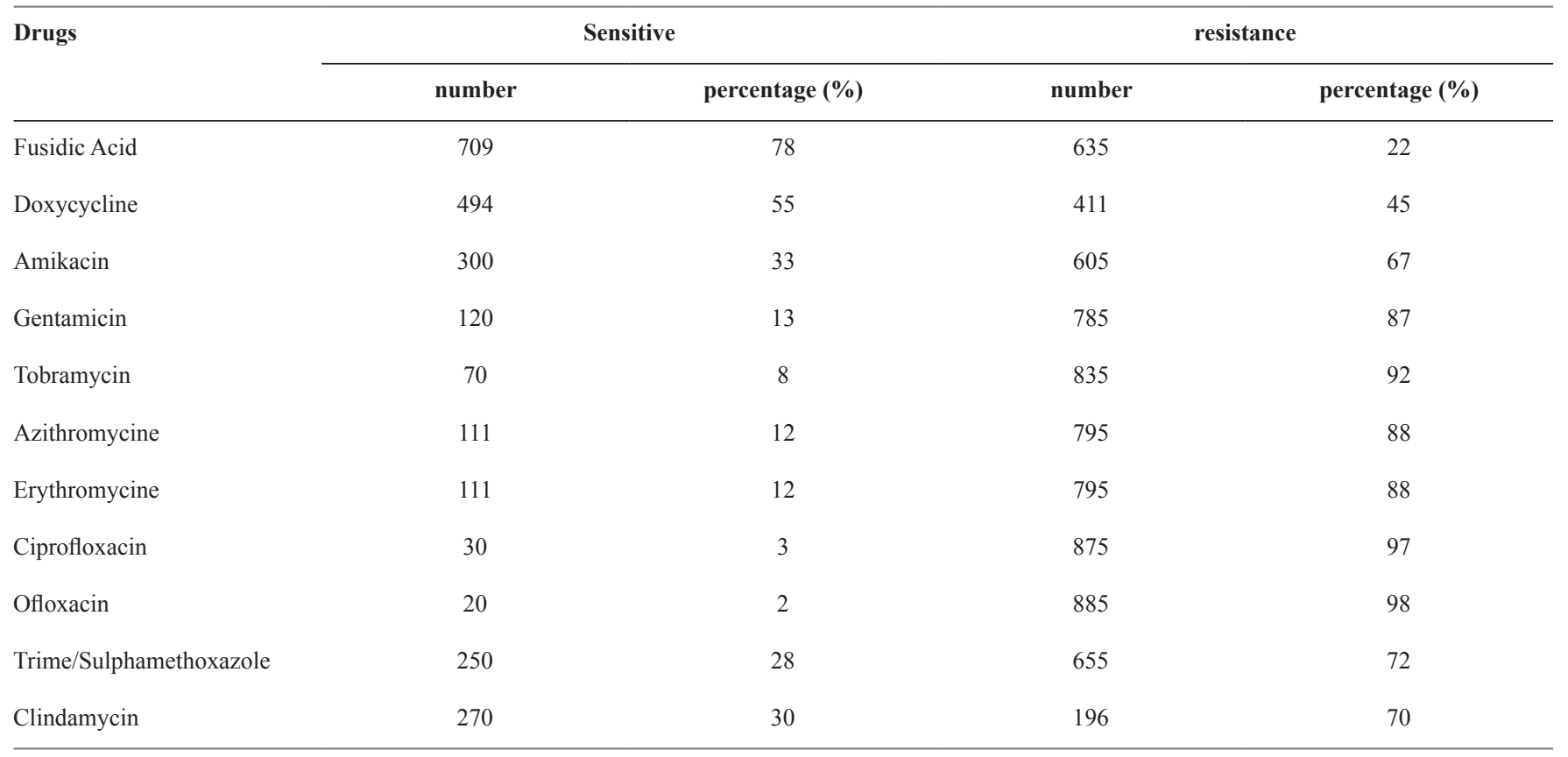

MRSA: methicillin resistant Staphylococcus aureus.
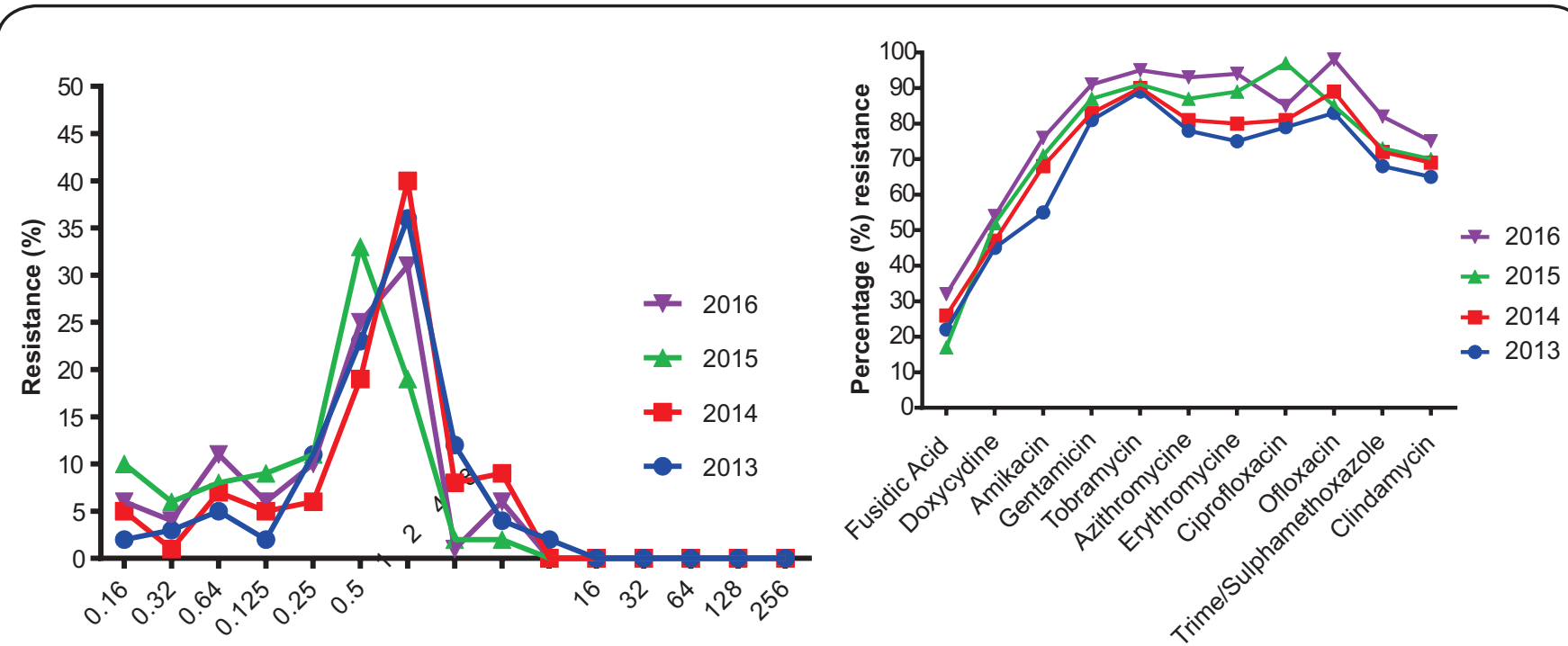

FIGURE 2 - Antibiotic resistance patterns of MRSA against different antibiotics isolated from different device-related infections. MRSA: methicillin resistant Staphylococcus aureus. 
In this study, the prevalence of bacterial pathogens causing device-related infection was determined and the recent trend in the antibiogram of MRSA was evaluated. The prevalence of prosthetic device-related infection was $76 \%$ which was in accordance with a study conducted in Turkey. The pathogens identified from prosthetic device-related infections were similar to many studies conducted in the past. E. coli (21\%) was the commonest cause of catheter-related infections, followed by MRSA (14.5\%) and K. pneumoniae (14.3\%). E. coli and Pseudomonas spp. were dominant in Europe while Streptococcus spp. and Candida spp. were the dominant causes of device-related infections in the USA, depending on the health care facility. The prevalent pathogens infecting CVCs were K. pneumoniae and Candida spp. (10\% each) followed by Streptococcus spp. (9\%), Pseudomonas spp. (7\%), E. coli (5\%) and Acinetobacter spp. (4\%); these prevalence rates are in accordance with the results reported in a study conducted from 2011 to $2013^{4}$. Our study also supported the retrospective survey conducted in Italy except for CONS which was reported at $34 \%$ and the result of our study showed $16 \%$; that retrospective study was conducted on oncological patients which were more prone to CONS infections ${ }^{5}$.

For catheter-related UTI, our results were similar to a retrospective study conducted in India except for E.coli, Klebsiella and Pseudomonas spp; our results were 71\%, 8\% and $3 \%$ as compared to $35 \%, 21 \%$ and $17 \%$, respectively ${ }^{6}$. This study was in agreement with the results of a study in Saudi Arabia in 2010 except for E. coli $(71 \%$ vs $40 \%)$. This difference was due to the varying demographic and health care settings. Different studies reported different prevalence rates of uropathogens, Candida spp. (28.2\%); K. pneumoniae (44\%) and $E$. coli $(70 \%)$; depending on the nature of sample, immune status and the underlying disease of the patient. E. coli was not only most common cause of urinary catheter-related infection, but most device related-infections were due to $E$. coli as claimed in the present study.

The prevalence of pathogens in ETT infection was not in agreement with a study published in the United States except for Klebsiella spp. because only VAP was included in the study? Yagmurdur reported the prevalence of Acinetobacter spp. (51\%) and Pseudomonas spp. (17\%); the prevalence of Acinetobacter spp. was greater because it was a major cause of $\mathrm{VAP}^{8}$ which was the theme of that study. K. pneumoniae (30\%) was the major cause of ETT infection followed by Acinetobacter spp. (25\%), followed by Pseudomonas spp. (22\%), Candida spp. and MRSA (7\% each), MSSA (4\%), E. coli and Streptococcus spp. (2\% each), and CONS ( $1 \%)$. The prevalence of pathogens isolated from ETTs was the same as that reported in a study conducted in Poland in 2014.

A study conducted in Iran demonstrated same the prevalence of pathogens especially MRSA. Orthopedic implant-related infections were worse and it is difficult to eradicate the causative pathogen because of low drug penetration in bone tissues. Staphylococcus spp. are notorious for causing bone and joint infections and form a biofilm on implants. Half (45-55\%) of bone and joint infections were due to $S$. aureus especially related to orthopedic implants. MRSA was the most common (30\%) cause of infected orthopedic implants, followed by CONS (20\%), MSSA and $K$. pneumoniae (15\% each), and Candida spp., Pseudomonas spp., Acinetobacter spp. and E. coli (5\% each). A previous study showed an increase in Acinetobacter spp. (16\%) isolated in orthopedic infections, while the prevalence of other pathogens was the same?.

Staphylococcus spp. (28\%) and Pseudomonas spp. (8\%) were the major causes of peritoneal dialysis catheter infection; similar results were obtained in Senegal and Sudan ${ }^{10}$. The results of this study strongly agreed with the results of a retrospective study conducted internationally. Shunt infections are caused by CONS (22\%), followed by Pseudomonas spp. (15\%) and E. coli $(12 \%)$. Similar results were obtained in a retrospective study conducted in Switzerland and Canada, whereas some other studies related to pediatric CSF reported Staphylococcus spp. as the most common cause of infection $(75 \%)^{11}$.

MRSA isolated from prosthetic devices showed a $67 \%$ resistance to amikacin and $87 \%$ resistance to gentamycin; these results disagreed with those of a previous study. The antibiogram of azithromycin (88\%), erythromycin (88\%), ciprofloxacin (97\%), trimethoprim/sulphamethoxazole $(71 \%)$ was quite similar to that reported in a study conducted in Nepal ${ }^{12}$; similar results were also reported in Iran except for fusidic acid ${ }^{13}$. Some studies reported a high prevalence of resistance to fusidic acid $(60 \%)$ and clindamycin $(71 \%)$ which strongly agreed with the results of the present study ${ }^{14}$. The resistance of MRSA to aminoglycosides was very high compared to a study conducted in Germany, which reported a $23 \%$ resistance to amikacin and $24 \%$ to gentamycin ${ }^{15}$. The susceptibility of vancomycin was almost $100 \%$ which strongly agreed with studies conducted in Spain, India, China, and Nepal. This study showed an MIC of $<16 \mathrm{ug} / \mathrm{ml}$ for vancomycin but some studies reported MIC an greater than $16 \mathrm{ug} / \mathrm{ml}$ as well ${ }^{16}$. In the present study the difference in the antibiogram was due to MRSA isolated from six different types of prosthetic device-related infection. Other studies considered one or two types of device-related infection. There was a gradual increase in the acquisition of resistance by MRSA as shown in Figure 2, but this difference in resistance was not statistically significant ( $\mathrm{p}>0.05)$.

Prosthetic device-related infections are a major concern today; due to advancements in medicine and surgery, prosthetic devices are used frequently for better health care facilities. This study demonstrated the prevalence of pathogens from six different types of prosthetic devices and catheters. This revealed the recent trend of antibiogram of MRSA which is emerging as a superbug of device related infections. This data will help clinicians to manage device-related infections effectively.

\section{Acknowledgements}

We highly acknowledge the managements of Chughtais Lab, Lahore for supporting this study.

\section{Financial support}

This study received funding from the Higher Education Commission (HEC), Pakistan under pin no. 112-22691-2BM1-376. 


\section{Conflict of interest}

The authors declare that there is no conflict of interest.

\section{REFERENCES}

1. Stevens V, Geiger K, Concannon C, Nelson RE, Brown J, Dumyati G. Inpatient costs, mortality and 30-day re-admission in patients with central-line-associated bloodstream infections. Clin Microbiol Infect. 2014;20(5):O318-24.

2. Mujais S. Microbiology and outcomes of peritonitis in North America. Kidney Int. 2006;70(Suppl 103):S55-62.

3. Clinical and Laboratory Standards Institute (CLSI). Performance Standards for Antimicrobial Susceptibility Testing: 26th Informational supplement. In: document M100-S26. Wayne, PA: CLSI; 2016.

4. Bouzad C, Duron S, Bousquet A, Arnaud FX, Valbousquet L, Weber-Donat G, et al. Peripherally inserted central catheter-related infections in a cohort of hospitalized adult patients. J Cardiovasc Intervent Radiol. 2016;39(3):385-93.

5. Wisplinghoff $\mathrm{H}$, Seifert H, Wenzel RP, Edmond MB. Current trends in the epidemiology of nosocomial bloodstream infections in patients with hematological malignancies and solid neoplasms in hospitals in the United States. Clin Infect Dis. 2003;36(9):1103-10.

6. Nandini MS, Madhusudan K. Bacteriological profile of catheter associated urinary tract infection and its antimicrobial susceptibility pattern in a tertiary care hospital. J Pharm Sci Res. 2016;8(4):204-7.

7. Apisarnthanarak A, Holzmann-Pazgal G, Hamvas A, Olsen MA, Fraser VJ. Ventilator-associated pneumonia in extremely preterm neonates in a neonatal intensive care unit: characteristics, risk factors, and outcomes. Pediatrics. 2003;112(6 Pt 1):1283-9.

8. Yagmurdur H, Tezcan AH, Karakurt O, Leblebici F. The efficiency of routine endotracheal aspirate cultures compared to bronchoalveolar lavage cultures in ventilator-associated pneumonia diagnosis. Niger J Clin Pract. 2016;19(1):46-51.

9. Helal S, El Anany M, Ghaith D, Rabeea S. The Role of MDRAcinetobacter baumannii in orthopedic surgical site infections. Surg Infect (Larchmt). 2015;16(5):518-22.

10. Niang A, Cisse MM, Mahmoud SMOM, Lemrabott ATO, Ka el HF, Diouf B. Pilot experience in senegal with peritoneal dialysis for end-stage renal disease. Perit Dial Int. 2014;34(5):539-43.

11. Odio C, McCracken Jr GH, Nelson JD. CSF shunt infections in pediatrics. A seven-year experience. Am J Dis Child. 1984;138(12):1103-8.

12. Bhatta DR, Cavaco LM, Nath G, Gaur A, Gokhale S, Bhatta DR. Threat of multidrug resistant Staphylococcus aureus in Western Nepal. Asian Pac J Trop Dis. 2015;5(8):617-21.

13. Moghadam SO, Pourmand MR, Aminharati F. Biofilm formation and antimicrobial resistance in methicillin-resistant Staphylococcus aureus isolated from burn patients, Iran. J Infect Dev Ctries. 2014;8(12):1511-7.

14. Girgis SA, El Din Gomaa HE, Saad NE, Salem MM. A comparative study for detection of methicillin resistance Staphylococci by polymerase chain reaction and phenotypic methods. Life Sci J. 2013;10(4):3711-8.

15. Hu Y, Liu A, Vaudrey J, Vaiciunaite B, Moigboi C, McTavish SM, et al. Combinations of $\beta$-Lactam or aminoglycoside antibiotics with plectasin are synergistic against methicillin-sensitive and methicillin-resistant Staphylococcus aureus. PLoS One. 2015;10(2):e0117664.

16. Hasan R, Acharjee M, Noor R. Prevalence of vancomycin resistant Staphylococcus aureus (VRSA) in methicillin resistant $S$. aureus (MRSA) strains isolated from burn wound infections. Ci Ji Yi Xue Za Zhi. 2016;28(2)49-53. 